\title{
Chemical Shift Anisotropy Measurements of Amide and Carbonyl Resonances in a Microcrystalline Protein with Slow Magic-Angle Spinning NMR Spectroscopy
}

Benjamin J. Wylie, Lindsay J. Sperling, Heather L. Frericks, Gautam J. Shah, W. Trent Franks, and Chad M. Rienstra*

Department of Chemistry, Department of Biochemistry and Center for Biophysics and Computational Biology, University of Illinois at Urbana-Champaign, 600 South Mathews Avenue, Urbana, Illinois 61801 E-mail: rienstra@scs.uiuc.edu

\section{Supporting Information}

\section{Experimental Procedures}

The $2 \mathrm{D}{ }^{15} \mathrm{~N}-{ }^{13} \mathrm{C}$ correlation experiments were executed on $750 \mathrm{MHz}$ Unity Inova spectrometer (Varian, Inc., Palo Alto, California) with $3.2 \mathrm{~mm}$ BioMAS $^{\mathrm{TM}}$ probe $^{1}$ optimized for ${ }^{1} \mathrm{H}-{ }^{13} \mathrm{C}-{ }^{15} \mathrm{~N}$ operation. This probe design offers especially high $\mathrm{B}_{1}$ homogeneity (i.e., $\mathrm{I}(810) /$ I(90) values in nutation profiles of $\sim 90 \%$ for all nuclei), and greatly reduced dielectric RF heating, which permitted the indirect ${ }^{15} \mathrm{~N}$ dimension to be digitized to approximately $20 \mathrm{~ms}$, for example with 900 complex points and a dwell of $22.2 \mu$ s for the $3 \mathrm{kHz}$ MAS data set. The direct ${ }^{13} \mathrm{C}$ dimension was digitized with 8,192 complex points and a bandwidth of $200 \mathrm{kHz}$ (maximum acquisition time of $40.96 \mathrm{~ms}$ ) with $\sim 80 \mathrm{kHz}$ TPPM decoupling. ${ }^{2}$ The $1,3-{ }^{13} \mathrm{C}$-glycerol $-{ }^{15} \mathrm{~N}-\mathrm{GB} 1$ sample was grown in minimal medium using $1,3{ }^{13} \mathrm{C}$-glycerol $(2 \mathrm{~g} / \mathrm{L})$ and $\mathrm{Na}^{12} \mathrm{CO}_{3}$ as the sole carbon sources and prepared by precipitation as described elsewhere. ${ }^{3}$ For this study, $\sim 18 \mathrm{mg}$ of protein was packed into the central $80 \%$ of the volume of a Varian limited speed $3.2 \mathrm{~mm}$ rotor. The MAS rates were controlled within $\pm 6 \mathrm{~Hz}$ or better over the course of each experiment.

\section{Analysis of Slow Magic-Angle Spinning (MAS) Data}

Slow MAS data was processed using NMRPipe. ${ }^{4}$ A Lorentzian-to-Gaussian apodization function was employed, with a net line broadening of 20 to $40 \mathrm{~Hz}$ in each of the two isotropic chemical shift dimensions, followed by zero filling and polynomial frequency-domain baseline correction. Each correlation manifold of the isotropic ${ }^{15} \mathrm{~N}-{ }^{13} \mathrm{C} 2 \mathrm{D}$ plane $(\mathrm{F} 1, \mathrm{~F} 2)$ was separately 
analyzed by modeling each peak to a Gaussian and integrating peak intensities using the autoFit.tcl and NMRWish packages provided with NMRPipe. ${ }^{4}$ This fitting was driven by inhouse shell scripts and FORTRAN programs which generate peak lists and fitting scripts from the centerband peak table. The $1 \mathrm{D}{ }^{15} \mathrm{~N}$ and ${ }^{13} \mathrm{C}$ manifolds were generated by summation along the ${ }^{13} \mathrm{C}$ and ${ }^{15} \mathrm{~N}$ dimensions respectively. The three manifolds for each resonance were fit simultaneously via a grid search to a library of SPINEVOLUTION simulations. ${ }^{5}$ Two libraries were generated for each manifold, one considering the effects of heteronuclear ${ }^{15} \mathrm{~N}-{ }^{13} \mathrm{C}^{\prime}$ dipolar and $\mathbf{J}$ couplings, and one neglecting these effects. It was found that inclusion of dipolar and $\mathbf{J}$ coupling terms did not improve the quality of the fit and had negligible impact on the value of the anisotropy parameters, due to the fact these inhomogeneous terms in the Hamiltonian are significantly smaller in magnitude than the spinning rates used here and that we did not attempt to determine de novo tensor orientations (to do so would require fits of the entire manifold of sidebands in both dimensions without calculating projections). In the convention of Haeberlen, ${ }^{6}$ a two-dimensional grid of simulated data was generated with $\delta_{\text {aniso }}$ incremented in steps of 0.25 ppm and $\eta$ incremented in steps of 0.025 from 0 to 1 , with linear interpolation of the trajectories between points on the grid. The fitting was conducted using a series of nested loops. Optimal agreement was judged by the minimal value for the root mean squared deviation (RMSD) between the experimental and simulated data. A Monte Carlo algorithm was implemented to determine standard deviations, in the same manner as described in our previous studies. ${ }^{7,8}$ In this analysis technique, Gaussian random noise was scaled by twice the RMSD of the best fit simulation and added to the experimental data. This procedure is repeated $1,000-10,000$ times, and the error bars given here are the standard deviation of this ensemble of fits.

\section{Differences in the ${ }^{15} \mathrm{~N}$ Tensor Magnitude from Previous Measurements}

Overall, the measurements of $\delta\left({ }^{15} \mathrm{~N}\right)$ are $\sim 10 \%$ larger than our previous study, ${ }^{7}$ which is explained by a small shift in the measured magnitude of $\delta_{y y}$. Our previous study was performed 
by a recoupling experiment (ROCSA) $)^{9}$ in which high power ${ }^{1} \mathrm{H}$ decoupling is especially critical for precise determination of the asymmetry parameter, $\eta$. As we reported in the previous work, this led to a systematic difference in this parameter between ROCSA and Herzfeld-Berger analysis. In the ROCSA experiments, the average value of $\eta$ was determined to be 0.385 and the average span, $\Omega\left(\Omega=\left|\delta_{z z}-\delta_{x x}\right|\right)$ was $172.6 \mathrm{ppm}$. Here, we find the average magnitude of $\Omega$ is $173.9 \mathrm{ppm}$, within experimental error. From this, it appears that the relaxation seen in the ROCSA $^{9}$ technique slightly distorts the magnitude of $\delta_{y y}$ for resonances where $\eta<0.5$, resulting in a small decrease in $\delta$ and a small increase in $\eta$. In addition, values of $\delta$ show greater dependence upon secondary structure compared to many solution studies. Unlike these studies, we did not adjust for molecular motions based upon order parameters. Because such order parameters are based upon scaling of dipolar tensors, it is not clear that such scaling is appropriate for measurements involving (essentially) only one spin. 
Supporting Table 1. Backbone Amide ${ }^{15}$ N CSA Tensor Values

\begin{tabular}{|c|c|c|c|c|}
\hline Residue $^{1}$ & $\delta($ ppm) & $\sigma_{\delta}(\mathrm{ppm})^{2}$ & $\eta$ & $\sigma_{\eta}$ \\
\hline $\mathbf{L 5}$ & -112.0 & 1.2 & 0.00 & 0.04 \\
\hline L7 & -103.5 & 1.4 & 0.13 & 0.13 \\
\hline G9 & -105.5 & 2.0 & 0.28 & 0.14 \\
\hline K10 & -111.5 & 2.0 & 0.00 & 0.13 \\
\hline T11 & -108.0 & 1.2 & 0.03 & 0.06 \\
\hline L12 & -112.0 & 2.5 & 0.20 & 0.15 \\
\hline G14 & -106.0 & 2.0 & 0.45 & 0.08 \\
\hline E15 & -110.5 & 1.8 & 0.10 & 0.13 \\
\hline T16 & -106.5 & 2.4 & 0.03 & 0.16 \\
\hline T17 & -105.3 & 2.3 & 0.38 & 0.15 \\
\hline T18 & -105.0 & 2.3 & 0.48 & 0.12 \\
\hline A20 & -109.5 & 1.7 & 0.33 & 0.13 \\
\hline V21 & -109.5 & 2.4 & 0.23 & 0.16 \\
\hline D22 & -117.5 & 1.6 & 0.00 & 0.11 \\
\hline T25 & -115.5 & 1.4 & 0.03 & 0.07 \\
\hline A26 & -115.2 & 2.2 & 0.30 & 0.14 \\
\hline E27 & -110.5 & 1.5 & 0.18 & 0.12 \\
\hline K28 & -112.8 & 2.1 & 0.43 & 0.10 \\
\hline K31 & -118.0 & 1.9 & 0.20 & 0.13 \\
\hline Y33 & -117.5 & 1.9 & 0.30 & 0.13 \\
\hline A34 & -117.5 & 1.9 & 0.13 & 0.11 \\
\hline N35 & -112.5 & 2.1 & 0.18 & 0.13 \\
\hline N37 & -106.5 & 1.8 & 0.33 & 0.13 \\
\hline G38 & -114.0 & 1.8 & 0.00 & 0.02 \\
\hline V39 & -113.5 & 1.4 & 0.00 & 0.05 \\
\hline D40 & -103.0 & 1.2 & 0.15 & 0.12 \\
\hline G41 & -85.0 & 1.5 & 0.03 & 0.15 \\
\hline E42 & -103.5 & 1.8 & 0.30 & 0.13 \\
\hline W43 & -106.5 & 2.0 & 0.18 & 0.10 \\
\hline T44 & -105.5 & 1.8 & 0.40 & 0.10 \\
\hline Y45 & -102.5 & 2.3 & 0.28 & 0.16 \\
\hline D46 & -107.0 & 1.5 & 0.03 & 0.08 \\
\hline D47 & -109.0 & 3.0 & 0.28 & 0.18 \\
\hline A48 & -115.0 & 2.1 & 0.30 & 0.15 \\
\hline T49 & -104.5 & 1.6 & 0.00 & 0.06 \\
\hline K50 & -116.5 & 1.8 & 0.03 & 0.10 \\
\hline T51 & -109.0 & 1.9 & 0.03 & 0.07 \\
\hline F52 & -111.0 & 2.5 & 0.00 & 0.13 \\
\hline T53 & -105.0 & 2.8 & 0.50 & 0.05 \\
\hline V54 & -108.5 & 2.4 & 0.50 & 0.06 \\
\hline T55 & -106.0 & 1.5 & 0.28 & 0.12 \\
\hline E56 & -112.0 & 1.8 & 0.00 & 0.14 \\
\hline
\end{tabular}

${ }^{1}$ Residues not reported include those following Leu, for which the ${ }^{13} \mathrm{C}^{\prime}$ site is not labeled, the amino terminal residue, and a small subset of other resonances that are not satisfactorily resolved in the slow MAS 2D spectra. ${ }^{2}$ The uncertainty is quoted at the $68 \%$ confidence interval. 


\section{Supporting Table 2. Backbone Carbonyl ${ }^{13} \mathrm{C}$ CSA Tensor Values}

\begin{tabular}{|c|c|c|c|c|}
\hline Residue $^{1}$ & $\delta(\mathbf{p p m})$ & $\sigma_{\delta}(\mathbf{p p m})^{2}$ & $\eta$ & $\sigma_{\eta}$ \\
\hline K4 & -77.3 & 2.3 & 0.73 & 0.04 \\
\hline I6 & -78.8 & 2.5 & 0.75 & 0.05 \\
\hline N8 & -76.5 & 2.1 & 0.60 & 0.05 \\
\hline G9 & -77.3 & 1.9 & 0.73 & 0.04 \\
\hline K10 & -80.8 & 1.9 & 0.48 & 0.05 \\
\hline T11 & -74.8 & 2.4 & 0.78 & 0.06 \\
\hline K13 & -76.8 & 1.7 & 0.78 & 0.03 \\
\hline G14 & -75.0 & 1.5 & 0.75 & 0.04 \\
\hline E15 & -78.5 & 3.2 & 0.78 & 0.07 \\
\hline T16 & -73.0 & 2.1 & 0.83 & 0.05 \\
\hline T17 & -79.3 & 2.1 & 0.70 & 0.05 \\
\hline E19 & -79.0 & 2.4 & 0.70 & 0.05 \\
\hline A20 & -83.5 & 2.0 & 0.55 & 0.05 \\
\hline V21 & -81.0 & 1.9 & 0.50 & 0.05 \\
\hline A24 & -85.8 & 1.9 & 0.43 & 0.05 \\
\hline T25 & -83.0 & 1.6 & 0.58 & 0.04 \\
\hline A26 & -83.8 & 2.5 & 0.55 & 0.06 \\
\hline E27 & -85.5 & 1.9 & 0.53 & 0.04 \\
\hline F30 & -84.3 & 2.3 & 0.50 & 0.05 \\
\hline Q32 & -81.3 & 2.1 & 0.48 & 0.06 \\
\hline Y33 & -84.8 & 1.3 & 0.50 & 0.03 \\
\hline A34 & -84.0 & 1.6 & 0.48 & 0.04 \\
\hline D36 & -79.0 & 2.4 & 0.70 & 0.05 \\
\hline N37 & -78.5 & 1.7 & 0.65 & 0.04 \\
\hline G38 & -78.0 & 1.8 & 0.68 & 0.04 \\
\hline V39 & -75.3 & 1.4 & 0.82 & 0.03 \\
\hline D40 & -72.3 & 2.3 & 0.80 & 0.05 \\
\hline G41 & -74.8 & 1.9 & 0.78 & 0.04 \\
\hline E42 & -82.0 & 2.3 & 0.53 & 0.06 \\
\hline W43 & -81.8 & 2.5 & 0.55 & 0.06 \\
\hline T44 & -77.3 & 2.1 & 0.73 & 0.05 \\
\hline Y45 & -76.8 & 1.6 & 0.83 & 0.04 \\
\hline D46 & -80.0 & 3.2 & 0.60 & 0.06 \\
\hline D47 & -81.3 & 1.8 & 0.55 & 0.04 \\
\hline A48 & -79.8 & 2.0 & 0.60 & 0.05 \\
\hline T49 & -80.3 & 1.9 & 0.63 & 0.04 \\
\hline K50 & -76.0 & 2.6 & 0.83 & 0.07 \\
\hline T51 & -81.5 & 2.8 & 0.60 & 0.06 \\
\hline F52 & -78.0 & 2.6 & 0.65 & 0.05 \\
\hline T53 & -77.3 & 2.6 & 0.85 & 0.05 \\
\hline V54 & -78.0 & 2.1 & 0.73 & 0.04 \\
\hline T55 & -78.8 & 1.9 & 0.65 & 0.05 \\
\hline
\end{tabular}

${ }^{1}$ Residues not reported include Leu, for which the ${ }^{13} \mathrm{C}^{\prime}$ site is not labeled, the carboxy and amino terminal residues, and a small subset of other resonances that are not satisfactorily resolved in the slow MAS spectra.

${ }^{2}$ The uncertainty is quoted at the $68 \%$ confidence interval. 


\section{$\mathrm{N}-\mathrm{C}^{\prime}$ 2D at $3 \mathrm{kHz}$ MAS}

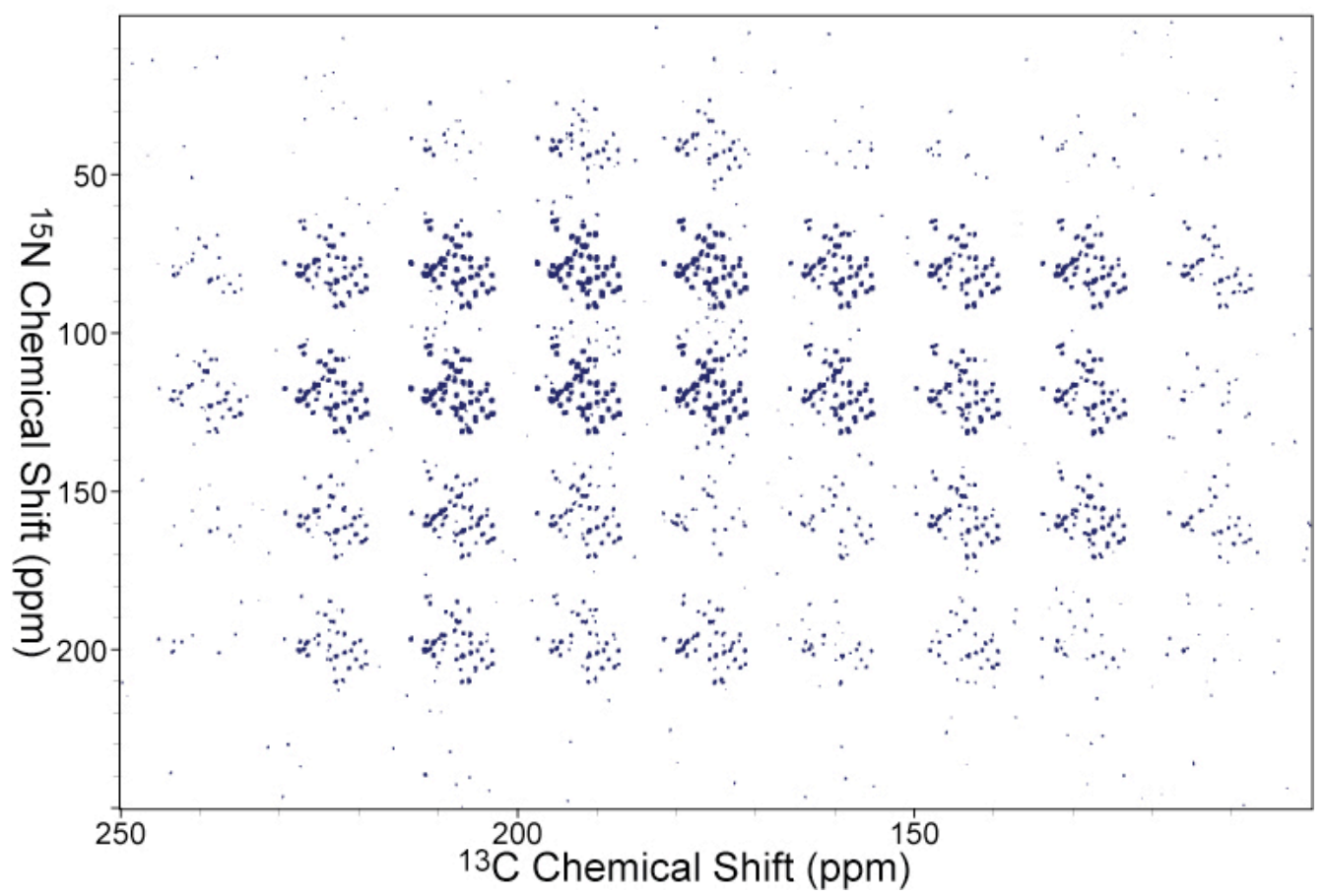

Figure S1. Slow 2D magic-angle spinning (MAS) ${ }^{15} \mathrm{~N}-{ }^{13} \mathrm{C}$ correlation spectrum of GB1, 750 $\mathrm{MHz}{ }^{1} \mathrm{H}$ frequency, at $3 \mathrm{kHz}$ MAS.

\section{$\mathrm{N}-\mathrm{C}^{\prime} 2 \mathrm{D}$ at $4 \mathrm{kHz}$ MAS}

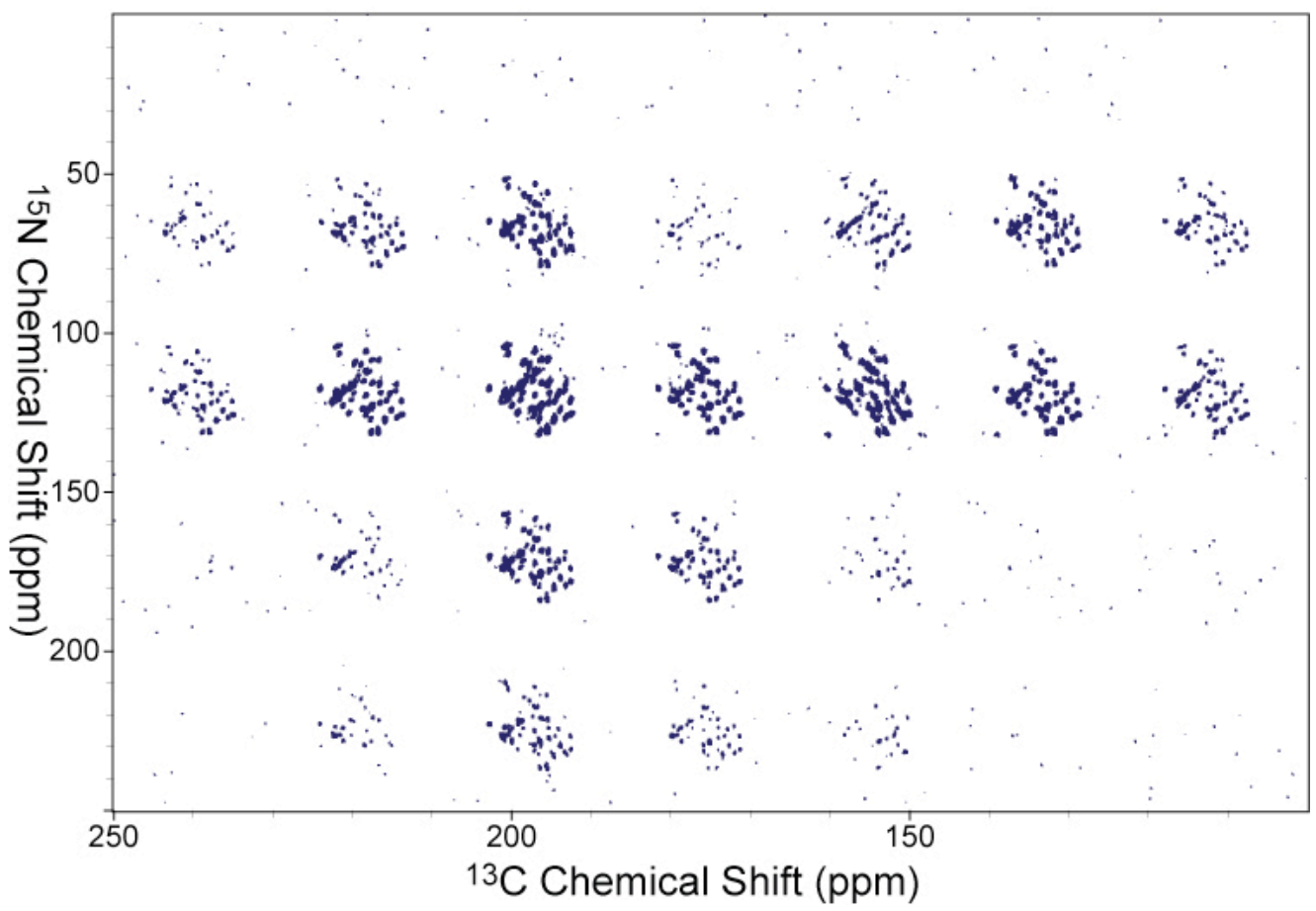

Figure S2. Slow 2D magic-angle spinning (MAS) ${ }^{15} \mathrm{~N}^{-13} \mathrm{C}$ correlation spectrum of GB1, 750 $\mathrm{MHz}{ }^{1} \mathrm{H}$ frequency, at $4 \mathrm{kHz}$ MAS. 


\section{$\mathrm{N}-\mathrm{C}^{\prime} 2 \mathrm{D}$ at $5 \mathrm{kHz}$ MAS}

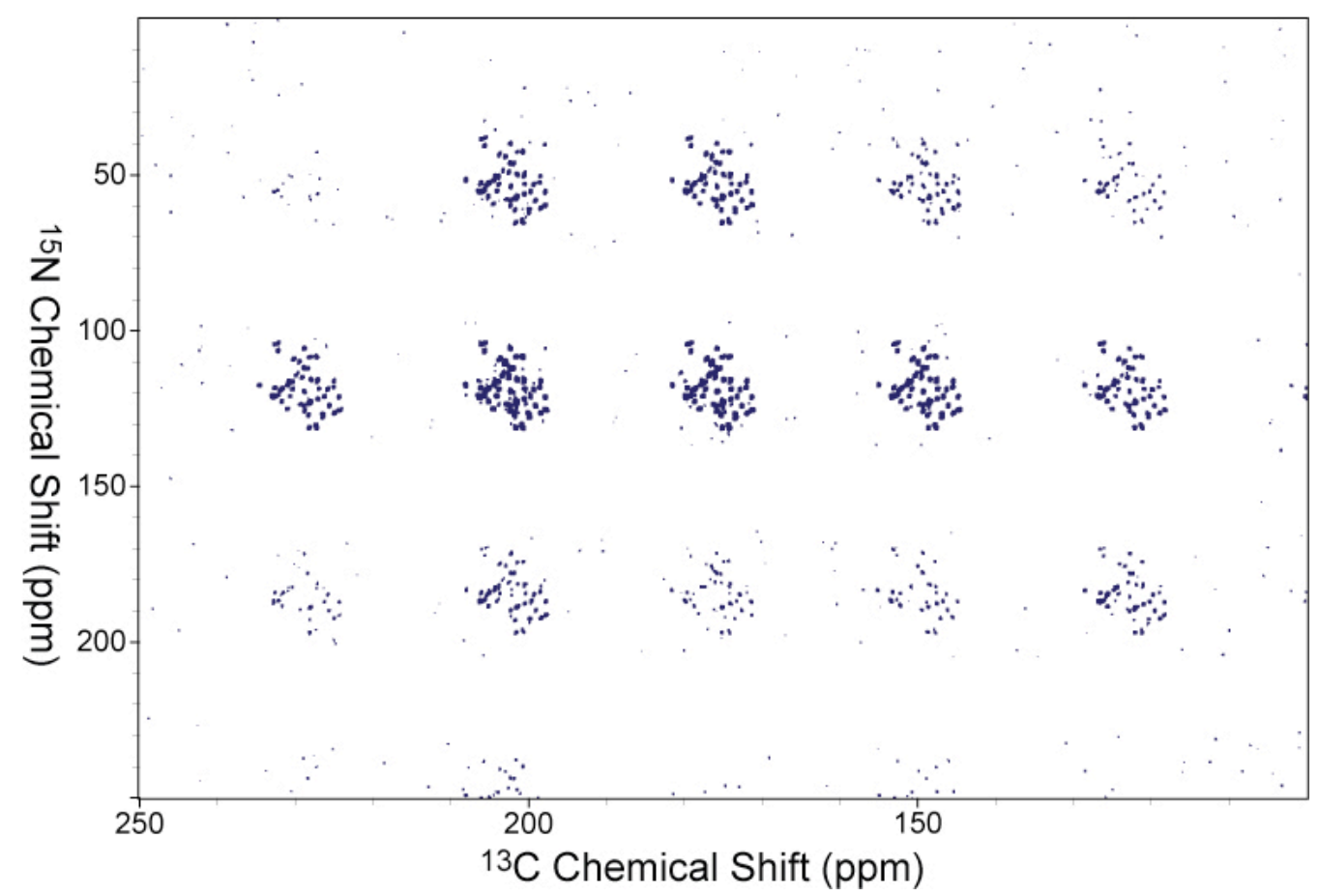

Figure S2. Slow 2D magic-angle spinning (MAS) ${ }^{15} \mathrm{~N}-{ }^{13} \mathrm{C}$ correlation spectrum of GB1, 750 $\mathrm{MHz}{ }^{1} \mathrm{H}$ frequency, at $5 \mathrm{kHz}$ MAS.

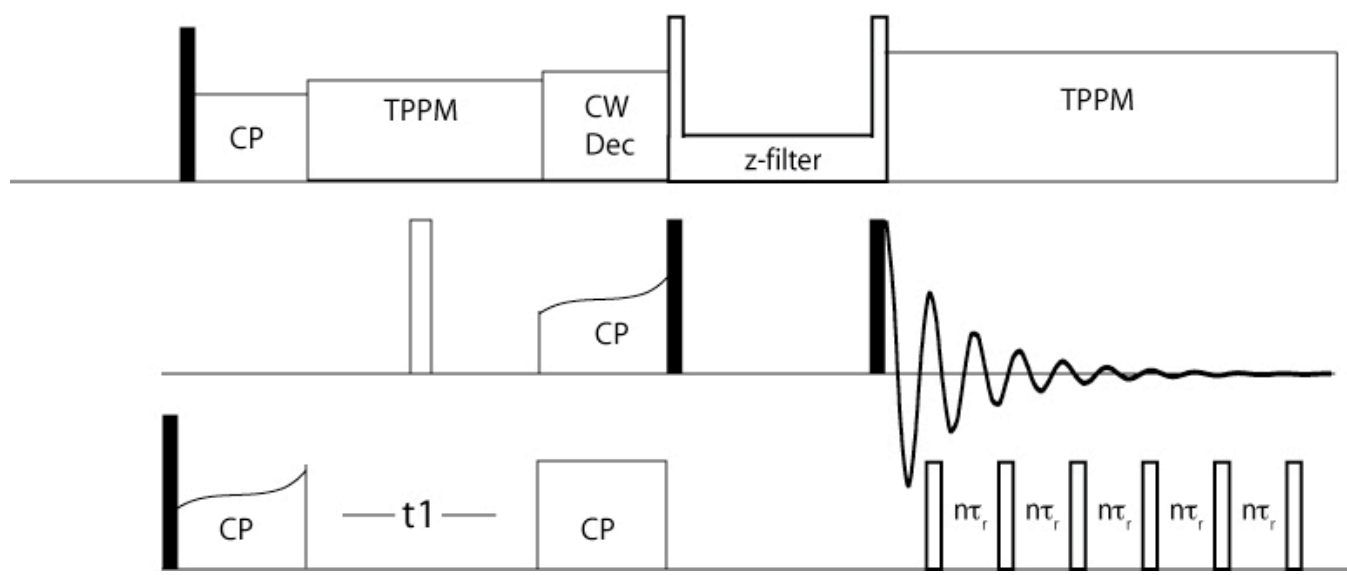

Figure S3. Pulse sequence employed for slow MAS experiments. Polarization is transferred from ${ }^{1} \mathrm{H}$ to ${ }^{15} \mathrm{~N}$ using adiabatic cross polarization, followed by a ${ }^{15} \mathrm{~N}$ chemical shift evolution period. After $\mathrm{t}_{1}$, polarization is transferred to ${ }^{13} \mathrm{C}$ using adiabatic SPECIFIC cross polarization. ${ }^{10}$ After a z-filter selects for the correct coherence order, ${ }^{13} \mathrm{C}$ is directly detected in $\mathrm{t}_{2}$ with the application of TPPM decoupling ${ }^{2}$ on ${ }^{1} \mathrm{H}$ and $\pi$ pulse decoupling on ${ }^{15} \mathrm{~N}$ using an XY-16 phase cycle. 


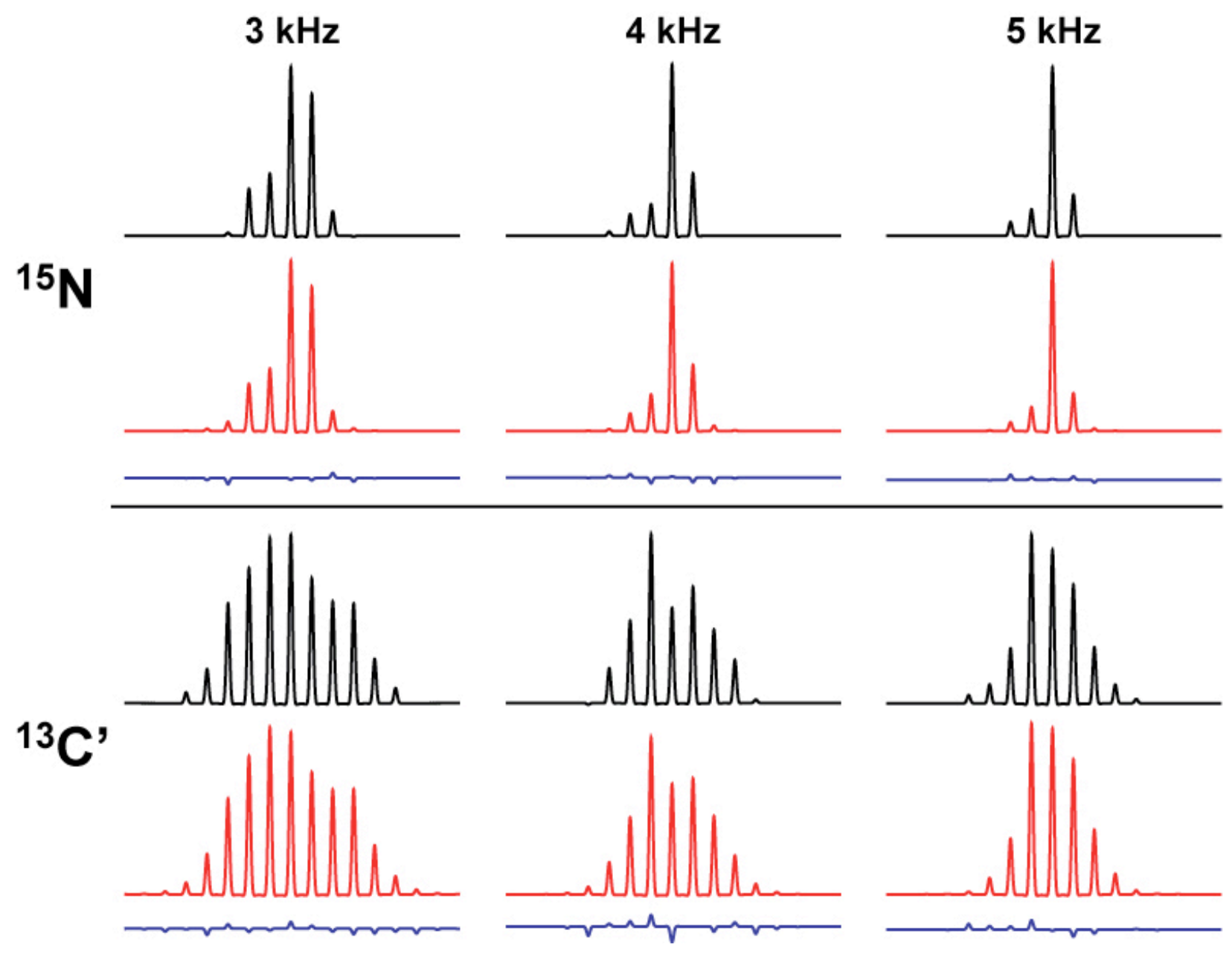

Figure S4. Expanded 1D sideband manifolds displayed in Figure 2 of main text. Spectra are reconstructed by summation of integrated intensities along one dimension. The D40N-V39C' site is illustrated here. Experimental intensity (black) is simulated (red) by adjusting $\mathrm{d}$ and h, but assuming canonical tensor orientations. ${ }^{3,4}$ Residuals are pictured in blue.

\section{References for Supporting Information:}

(1) Stringer, J. A.; Bronnimann, C. E.; Mullen, C. G.; Zhou, D. H. H.; Stellfox, S. A.; Li, Y.; Williams, E. H.; Rienstra, C. M. J. Magn. Reson. 2005, 173, 40-48.

(2) Bennett, A. E.; Rienstra, C. M.; Auger, M.; Lakshmi, K. V.; Griffin, R. G. J. Chem. Phys. 1995, 103, 6951-6958.

(3) Franks, W. T.; Zhou, D. H.; Wylie, B. J.; Money, B. G.; Graesser, D. T.; Frericks, H. L.; Sahota, G.; Rienstra, C. M. J. Am. Chem. Soc. 2005, 127, 12291-12305.

(4) Delaglio, F.; Grzesiek, S.; Vuister, G. W.; Zhu, G.; Pfeifer, J.; Bax, A. J. Biomol. NMR 1995, 6, 277-293.

(5) Veshtort, M.; Griffin, R. G. J. Magn. Reson. 2006, 178, 248-282.

(6) Haeberlen, U. High Resolution NMR in Solids: Selective Averaging; Academic Press: New York, 1976.

(7) Wylie, B. J.; Franks, W. T.; Rienstra, C. M. J. Phys. Chem. B 2006, 110, 10926-10936.

(8) Wylie, B. J.; Franks, W. T.; Graesser, D. T.; Rienstra, C. M. J. Am. Chem. Soc. 2005, 127, 11946-11947.

(9) Chan, J. C. C.; Tycko, R. J. Chem. Phys. 2003, 118, 8378-8389.

(10) Baldus, M., Petkova, A. T., Herzfeld, J. H., and Griffin, R. G. Mol. Phys. 1998, 95, 1197-1207. 